\section{CHEMISTRY}

\section{The hunt for explosives}

A dye-based sensor can detect tiny amounts of an explosive that has been used in several terrorist incidents.

Current methods for detecting triacetone triperoxide (TATP) have several drawbacks, such as being cumbersome or expensive. Kenneth Suslick and Hengwei Lin at the University of Illinois at Urbana-Champaign have developed a way to sense TATP levels as low as 2 parts per billion. They show that, in a gas flow treated with a solid acid catalyst, TATP decomposes into products, such as hydrogen peroxide, that can be detected with a colorimetric sensor.

The researchers have created a prototype hand-held detector that could be used to screen luggage. Importantly, the detector is not activated by other common compounds such as soaps, liquors or volatile organics.

J. Am. Chem. Soc. doi:10.1021/ ja107419t (2010)

\section{PHYSICS}

\section{Insulator insight} into constant

Enigmatic materials that conduct electricity at only their surfaces, known as topological insulators, could be used to measure the fine structure constant, $\alpha$ - one of three factors that determine the speed of light.

Joseph Maciejko at Stanford University in California and his team propose measuring $\alpha$ by observing the quantized magnetoelectric effect (QME) - a predicted phenomenon in which an electric field induces magnetization in discrete quantum steps. The proposed experiment would use a layer of a topological insulator on top of a layer of ordinary insulator, all sitting in an external magnetic field. The authors say that measuring the polarization of light reflected off the surface of the topological insulator, and comparing this with the measurement of the polarization of light transmitted through the layers, will reveal a measurement of the QME - and hence $\alpha$ - in a way that is independent of the materials' properties.

Phys. Rev. Lett. 105, 166803 (2010)

\section{CELL BIOLOGY}

\section{Spindle-free division in yeast}

During cell division, or mitosis, protein microtubules called spindles pull the replicated chromosomes apart before the cell splits in two. Stefania Castagnetti at Cancer Research UK in London and her group show that some yeast missing these spindles undergo a novel form of nuclear division - which may be a primitive form of mitosis.

Schizosaccharomyces pombe strains treated with a chemical that breaks down microtubules could still separate their chromosomes. By probing individual parts of the mitotic apparatus, the researchers surmise that, in the absence of spindles, the chromosomes remain associated with the cell's two spindle pole bodies, which normally act as anchors for the spindles. The authors suggest that these organelles move away from each other within the nuclear membrane, carrying the chromosomes along with them, before the nucleus divides.

PLoS Biol. 8, e1000512 (2010)

$$
\text { ECOLOGY }
$$

\section{What mammoths left behind}

Mass extinction of most of the world's large mammals some 10,000 years ago liberated roughly 1.4 petagrams of plant life previously consumed as food. The surplus endured until human populations grew to fill the void.

Christopher Doughty, now at the University of Oxford, UK, and Christopher Field at the Carnegie Institution in

COMMUNITY CHOICE

The most viewed papers in science

MOLECULAR BIOLOGY

\title{
Long RNAs turn up gene expression
}

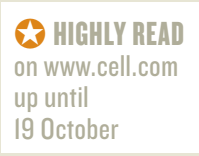

Long RNA molecules that do not code for proteins boost the expression of certain human genes, including those linked to development. Typically, regulatory RNAs, such as microRNAs, quiet gene expression.

Ramin Shiekhattar at the Wistar Institute in Philadelphia, Pennsylvania, and his colleagues found 3,019 RNA molecules, averaging 800 nucleotides in length, after scouring a portion of the human genome. When the team stimulated the development of a type of human skin cell, expression levels of many of the long non-coding RNA molecules rose in step with those of nearby protein-coding genes. Reducing the levels of a set of the RNA molecules in various cell lines also curbed the expression of neighbouring genes, including one coding for a protein that regulates blood-cell development. Cell 143, 46-58(2010)

Stanford, California, estimated consumption by the extinct Pleistocene megafauna and by humans, and compared the results with net primary plant production around the globe. Averaging the figures out worldwide, they found that liberated plant resources - about $2.5 \%$ of net terrestrial productivity - had been used up by humans by about 1700 .

The duo also showed that by 2000 , humans were consuming roughly six times more than the megafauna had done. Meanwhile, human agriculture had reduced global primary productivity by about $10 \%$ as a result of factors such as land degradation.

Environ. Res. Lett. 5, 044001 (2010)

\section{BIOCHEMISTRY}

\section{Zooming in on proteins}

High-resolution optical imaging of single molecules has been achieved in living cells through the design of a small fluorescent organic molecule that outperforms commonly used fluorescent proteins.

Organic fluorophores generally emit much more light than fluorescent proteins.
William Moerner of Stanford University in California and his colleagues have devised a system in which a commercial enzyme is fused to the protein of interest. The 'fluorogen' then binds to the enzyme and is activated by light, enabling high-resolution imaging by the controlled activation of single molecules.

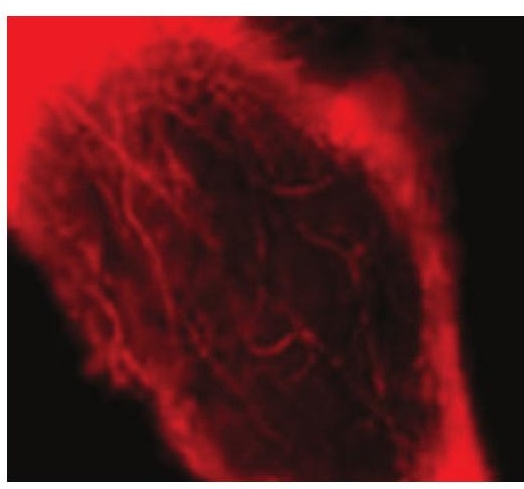

The authors were able to image protein microtubules in mammalian cells (pictured), as well as other protein structures in living bacteria, with a resolution beyond the limit of optical diffraction.

J. Am. Chem. Soc. doi:10.1021/ ja1044192 (2010)

\section{DNATURE.COM}

For the latest research published by Nature visit: www.nature.com/latestresearch 